\title{
Near-zero-energy end states in topologically trivial spin-orbit coupled superconducting nanowires with a smooth confinement
}

\author{
G. Kells, D. Meidan, and P. W. Brouwer \\ Dahlem Center for Complex Quantum Systems and Fachbereich Physik, Freie Universität Berlin, Arnimallee 14, 14195 Berlin, Germany
}

(Received 13 July 2012; revised manuscript received 3 September 2012; published 12 September 2012)

\begin{abstract}
A one-dimensional spin-orbit coupled nanowire with proximity-induced pairing from a nearby $s$-wave superconductor may be in a topological nontrivial state, in which it has a zero-energy Majorana bound state at each end. We find that the topological trivial phase may have fermionic end states with an exponentially small energy, if the confinement potential at the wire's ends is smooth. The possible existence of such near-zero-energy levels implies that the mere observation of a zero-bias peak in the tunneling conductance is not an exclusive signature of a topological superconducting phase, even in the ideal clean single channel limit.
\end{abstract}

DOI: 10.1103/PhysRevB.86.100503

PACS number(s): 74.78.Na, 03.67.Lx, 73.63.Nm, 74.20.Rp

In one dimension, topological superconducting wires are predicted to support a localized Majorana bound state at each end. ${ }^{1}$ These Majorana states are particle-hole symmetric and have exactly zero excitation energy. Within the associated degenerate subspace, braiding and exchange operations can be shown to be non-Abelian, ${ }^{2-4}$ making them potentially useful in topological quantum computation schemes. ${ }^{5-8}$ It has been recently noted that in the right parameter regime, spin-orbit coupled semiconductor nanowires with proximity-induced superconductivity should exhibit the required topological superconductivity for Majorana pair formation..$^{9,10}$

In light of these proposals, the experimental observations of zero-bias peaks in normal-metal superconductor tunnel junctions, which are unaffected by small variations of the magnetic field or gate voltages, may indicate the presence of topological superconductivity. ${ }^{11,12}$ While these observations are a necessary indicator of the predicted midgap Majorana states, ${ }^{13,14}$ it is crucial that alternative mechanisms for the zero-bias conductance be ruled out in order for them to be decisive. One example of such an alternative mechanism applies to quasi-one-dimensional wires with multiple conducting channels, ${ }^{15-19}$ for which low-energy fermionic bound states are predicted to appear in the topological as well as in the nontopological phase if the Zeeman energy exceeds the splitting between transverse subbands. ${ }^{20-24}$ Other alternative mechanisms involve disorder, ${ }^{25,26}$ possibly in combination with a gapless region at the wire's end. ${ }^{27}$ These latter findings suggest that clean single-channel wires offer a favorable setting to discern the presence of Majorana end states. Indeed, experiments are progressively approaching this ideal scenario. ${ }^{11,12}$

In this Rapid Communication we show that the original proposals $^{9,10}$ for topological superconductivity in clean onedimensional semiconductor wires also allow for near-zeroenergy end states deep in the topologically trivial phase, provided the potential that confines the electrons at the wire's end is smooth. The low energy is a systematic property of these states that persists as long as the confining potential and the induced superconductivity are smooth functions of position. The existence of such low-energy Andreev states leads to a low-energy peak in the tunneling conductance in the topologically trivial phase. Since gate-induced confinement potentials are typically smooth, the mechanism we describe here may be relevant for the recent experiments. ${ }^{11,12}$ Our analysis is consistent with and explains the observation of zero-bias conductance peaks in recent numerical simulations of clean semiconductor wires by Prada et al. ${ }^{28}$

Following the original theoretical proposals, ${ }^{9,10}$ we consider a one-dimensional semiconductor with a Rashba spinorbit coupling of strength $\alpha$, subject to a magnetic field with Zeeman energy $B>0$ and proximity coupled to a standard $s$ wave spin-singlet superconductor. Such a system is described by the four-component Bogoliubov-de Gennes Hamiltonian

$$
H=\left(\frac{p^{2}}{2 m}+V(x)-\mu-B \sigma_{x}+\alpha p \sigma_{y}\right) \tau_{z}+\Delta \sigma_{y} \tau_{x},
$$

where $\sigma_{x, y, z}$ and $\tau_{x, y, z}$ are Pauli matrices acting on the spin and particle-hole degrees of freedom, respectively. Further, $m$ is the effective electron mass, $\mu=p_{\mathrm{F}}^{2} / 2 m$ the chemical potential, $\Delta$ the proximity-induced superconducting gap in the absence of the magnetic field, and $V(x)$ is the potential that describes the confinement of electrons near the wire's end.

As shown in Refs. 9 and 10, the Hamiltonian (1) is in a topological phase with Majorana fermions at its ends if $B>$ $B_{\mathrm{c}}=\sqrt{\mu^{2}+\Delta^{2}}$. Here we consider the topologically trivial regime with weak induced superconductivity, $\Delta \ll B \ll \mu$. The condition $B \gg \Delta$ rules out spin-singlet $s$-wave pairing, so that the induced superconductivity must be of $p$-wave type. However, unlike in the topological regime, where the model (1) effectively admits $p$-wave superconductivity for one spin channel only, in the nontopological regime $B \ll \mu$ both spin channels acquire superconducting correlations. If $B \gg \Delta$ the two spin channels exist as effectively independent $p$-wave superconductors in the wire's bulk, but they are coupled at the wire's ends, which gaps out the pair of Majorana-like excitations that would have existed at the wire's end for uncoupled channels. As we show below, this coupling is strong if the wire's end is abrupt, but weak if the confinement is smooth, which explains the appearance of an Andreev bound state at an energy far below the bulk excitation gap. The crucial difference between an abrupt ending and a smooth confinement is that the spin-orbit energy $\varepsilon_{\mathrm{so}}=\alpha p$ remains finite up to the turning point for a hard-wall confinement, whereas $\varepsilon_{\text {so }}$ goes to zero continuously for a smooth confinement.

In order to arrive at an approximate analytical solution of this problem, we assume that the energies $B, \varepsilon_{\mathrm{so}}$, and 
$\Delta$ are much smaller than the kinetic energy $\mu-V(x)$. This separation of energy scales breaks down near the turning point at the wire's end, where the velocity

$$
v(x)=\sqrt{2[\mu-V(x)] / m}
$$

goes to zero. We circumvent this difficulty by solving a modified version of the problem, in which the wire has a hard-wall confinement with $V=0$ inside the wire, and a position-dependent spin-orbit strength $\tilde{\alpha}$ with

$$
\varepsilon_{\mathrm{So}}(x)=m \alpha v(x)=\tilde{\alpha}(\tilde{x}) p_{\mathrm{F}}
$$

to account for the position dependence of $\varepsilon_{\mathrm{so}}$. The two descriptions are essentially equivalent if the coordinate $\tilde{x}$ in the hard-wall model is related to the original coordinate $x$ as

$$
\tilde{x}=\int_{x_{0}}^{x} d x^{\prime} \frac{v_{\mathrm{F}}}{v(x)},
$$

where $v_{\mathrm{F}}=\sqrt{2 \mu / m}$ is the Fermi velocity in the hard-wall model. Taken together, the relations (3) and (4) ensure that the electrons "see" the same Zeeman energy $B$ and spin-orbit energy $\varepsilon_{\text {so }}$ as a function of time when they reverse their direction at the wire's end.

The inequalities $B, \varepsilon_{\mathrm{so}}, \Delta \ll \mu$ allow us to linearize the kinetic energy, writing

$$
\psi(\tilde{x})=\psi_{+}(\tilde{x}) e^{i p_{\mathrm{F}} \tilde{x} / \hbar}+\psi_{-}(\tilde{x}) e^{-i p_{\mathrm{F}} \tilde{x} / \hbar},
$$

where the functions $\psi_{ \pm}$are slow functions of position on the scale $\hbar / p_{\mathrm{F}}$. The function $\psi_{+}$describes right-moving electrons and left-moving holes, while $\psi_{-}$describes leftmoving electrons and right-moving holes. They are subject to the four-component Bogoliubov-de Gennes Hamiltonian

$$
\tilde{H}_{ \pm}=\mp i \hbar v_{\mathrm{F}} \tau_{z} \partial_{\tilde{x}}-B \sigma_{x} \tau_{z} \pm \tilde{\alpha}(\tilde{x}) p_{\mathrm{F}} \sigma_{y} \tau_{z}+\Delta \sigma_{y} \tau_{x},
$$

and the boundary condition $\psi_{+}(0)=e^{2 i \eta} \psi_{-}(0), \eta$ being a phase shift characteristic of the detailed boundary conditions at the wire's end at $\tilde{x}=0$.

The normal part of the Hamiltonian (6) can be diagonalized by a rotation in spin space. Defining the angle $\theta(\tilde{x})$ and the wave number $\tilde{k}_{\mathrm{m}}(\tilde{x})>0$ as

$$
\begin{aligned}
B & =\hbar v_{\mathrm{F}} \tilde{k}_{\mathrm{m}}(\tilde{x}) \cos \theta(\tilde{x}), \\
\varepsilon_{\mathrm{so}}(\tilde{x}) & =\hbar v_{\mathrm{F}} \tilde{k}_{\mathrm{m}}(\tilde{x}) \sin \theta(\tilde{x}),
\end{aligned}
$$

a basis change maps the Bogoliubov-de Gennes Hamiltonian (6) to $\tilde{H}_{0, \pm}+\tilde{H}_{1, \pm}$, with

$$
\begin{gathered}
\tilde{H}_{0, \pm}=\hbar v_{\mathrm{F}}\left(\mp i \partial_{\tilde{x}}-\tilde{k}_{\mathrm{m}} \sigma_{z}\right) \tau_{z} \pm \Delta \sigma_{z} \tau_{x} \sin \theta(\tilde{x}), \\
\tilde{H}_{1, \pm}=\Delta \sigma_{y} \tau_{x} \cos \theta(\tilde{x})+\frac{\hbar v_{\mathrm{F}}}{2} \frac{\partial \theta}{\partial \tilde{x}} \sigma_{x} \tau_{x} .
\end{gathered}
$$

The superconducting pairing in $\tilde{H}_{0, \pm}$ is of $p$-wave type and pairs electrons of equal spin (in the rotated frame) with a $p$-wave gap

$$
\Delta_{p}=\Delta \sin \theta=\frac{\varepsilon_{\mathrm{so}} \Delta}{\sqrt{B^{2}+\varepsilon_{\mathrm{so}}^{2}}},
$$

whereas the superconducting pairing in $\tilde{H}_{1, \pm}$ is of $s$-wave type and connects electrons of opposite spin.
In the limit $B \gg \Delta$ and for a smooth confining potential, $\tilde{H}_{1, \pm}$ can be treated in perturbation theory. The unperturbed Hamiltonian $\tilde{H}_{0, \pm}$ admits two zero-energy end states of Majorana type,

$$
\begin{aligned}
& \psi_{\uparrow, \pm}=\frac{e^{ \pm i \eta}}{\sqrt{\tilde{\Omega}}}\left(\begin{array}{c}
e^{-i \pi / 4} \\
0 \\
e^{i \pi / 4} \\
0
\end{array}\right) e^{\int_{0}^{\tilde{x}} d x^{\prime}\left[ \pm i \tilde{k}_{\mathrm{m}}\left(x^{\prime}\right)-1 / \tilde{\xi}\left(x^{\prime}\right)\right]}, \\
& \psi_{\downarrow, \pm}=\frac{e^{ \pm i \eta}}{\sqrt{\tilde{\Omega}}}\left(\begin{array}{c}
0 \\
e^{i \pi / 4} \\
0 \\
e^{-i \pi / 4}
\end{array}\right) e^{\int_{0}^{\tilde{x}} d x^{\prime}\left[\mp i \tilde{k}_{\mathrm{m}}\left(x^{\prime}\right)-1 / \tilde{\xi}\left(x^{\prime}\right)\right]},
\end{aligned}
$$

where the superconducting coherence length $\tilde{\xi}$ is defined as $\hbar v_{\mathrm{F}} / \tilde{\xi}(\tilde{x})=\Delta|\sin \theta(\tilde{x})|$ and $\tilde{\Omega}$ is a normalization constant. Calculating the matrix element of $\tilde{H}_{1, \pm}$ between these states, we find that the wire's end harbors a single Andreev end state with energy

$$
\begin{aligned}
\varepsilon= & \frac{2}{\tilde{\Omega}} \mid \int_{0}^{\infty} d \tilde{x}\left[2 \Delta \cos \theta(\tilde{x})+\hbar v_{\mathrm{F}} \frac{\partial \theta}{\partial \tilde{x}}\right] \\
& \times \cos \left[2 \int_{0}^{\tilde{x}} d x^{\prime} \tilde{k}_{\mathrm{m}}\left(x^{\prime}\right)\right] e^{-2 \int_{0}^{\tilde{x}} d x^{\prime} 1 / \tilde{\xi}\left(x^{\prime}\right)} \mid .
\end{aligned}
$$

Returning to the parameters of the original model (1), the energy $\varepsilon$ of the Andreev end state reads

$$
\begin{aligned}
\varepsilon= & \frac{2 B}{\Omega} \mid \int_{x_{0}}^{\infty} d x \frac{2 \Delta \sqrt{B^{2}+\varepsilon_{\mathrm{so}}(x)^{2}}-\hbar \alpha(d V / d x)}{v(x)\left[B^{2}+\varepsilon_{\mathrm{so}}(x)^{2}\right]} \\
& \times \cos \left[2 \int_{x_{0}}^{x} d x^{\prime} k_{\mathrm{m}}\left(x^{\prime}\right)\right] e^{-2 \int_{x_{0}}^{x} d x^{\prime} 1 / \xi\left(x^{\prime}\right)} \mid,
\end{aligned}
$$

where $\hbar v(x) k_{\mathrm{m}}(x)=m \alpha \xi(x) \Delta / \hbar=\sqrt{B^{2}+\varepsilon_{\text {so }}^{2}}$ and

$$
\Omega=4 \int_{x_{0}}^{\infty} d x \frac{e^{-2 \int_{x_{0}}^{x} d x^{\prime} 1 / \xi\left(x^{\prime}\right)}}{v(x)} .
$$

For the simple example that $V(x)$ has a linear dependence on $x$ near the wire's end, $V(x)=\mu-V^{\prime} x$, with the condition that $V^{\prime} \ll B \Delta / \hbar \alpha$, which ensures that $\varepsilon_{\text {so }} \ll B$ throughout the entire integration range, a closed-form expression can be obtained and one finds

$$
\varepsilon \approx \Delta e^{-B^{3} /\left(\hbar \alpha V^{\prime} \Delta\right)} .
$$

This result should be compared with the energy of the Andreev end state for a hard wall, which is

$$
\varepsilon=\left(\frac{\Delta^{2}}{2 B^{2}}+\frac{\varepsilon_{\mathrm{so}}}{\Delta}\right) \Delta
$$

if $\varepsilon_{\text {so }}, \Delta \ll B$. The energy (15) is essentially zero-even if compared with the $p$-wave gap $\Delta_{p}$-for a range of magnetic field far below the critical field $B_{\mathrm{c}}$ at which the transition to the topological phase takes place. (Incidentally, even with hard-wall boundary conditions, the Andreev end-state energy $\varepsilon$ may be small in comparison to $\Delta_{p}$ if $B$ is sufficiently large in comparison to $\varepsilon_{\text {so }}$ and $\Delta$.) Experimentally, the difference between the finite excitation energy $\varepsilon$ of Eq. (15) and the strict 
zero energy of the Majorana bound states may be difficult to resolve.

Both the splitting from the singlet pairing [first term in Eq. (9)] and the splitting from the nonadiabaticity of the confining potential [second term in Eq. (9)] vanish exponentially in the limit of a smooth, adiabatic confinement, provided the Zeeman energy $B$ sufficiently far exceeds $\Delta$. This is the main result of this Rapid Communication.

In the limit of an extremely small slope of the confining potential $V(x)$ there is another, trivial, effect, that also leads to the formation of near-zero-energy Andreev bound states: In principle, the smooth confinement leads to a spatial separation between the turning points for electrons of opposite spin, the region between the turning points being "topological." When the coherence length is shorter than the distance between the turning points, this topological segment hosts Majorana modes at its ends. These Majorana modes are spatially well separated and, hence, have an exponentially small energy that decreases with increasing $\Delta$. This is, however, not the situation we discuss here. Indeed, in the weak pairing limit, the energy (15) increases upon increasing $\Delta$, whereas the wave function of the Andreev end state extends over a length much larger than the distance between the classical turning points. (Note that this distance is zero in the effective hard-wall model used for our calculation.)

We have compared the theoretical predictions to numerical tight-binding simulations of a discretized version of the Hamiltonian (1). For this purpose we choose the confining potential

$$
V(x)= \begin{cases}a e^{-x^{2} / 2 \sigma^{2}}, & x>0, \\ \infty, & x<0,\end{cases}
$$

where the parameter $\sigma$ controls the degree of adiabaticity. Figures 1 and 2 show representative results of the numerical calculation, together with the analytical prediction of Eq. (13).

As discussed above, the small energy $\varepsilon$ of the Andreev end states results from the ineffectiveness of a smooth potential to

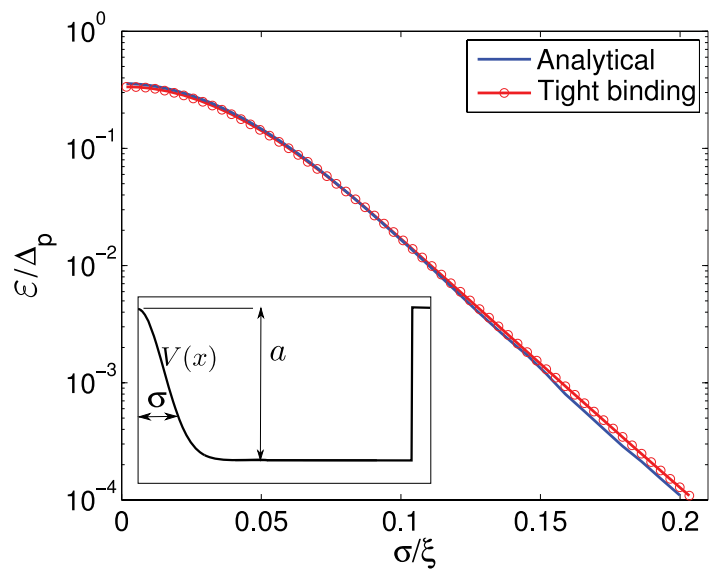

FIG. 1. (Color online) Andreev end-state energy $\varepsilon$, normalized to the bulk excitation gap $\Delta_{p}$, as a function of adiabaticity parameter $\sigma$. The red circles are obtained from a numerical calculation for which the wire is terminated by a smooth potential $V(x)$ of the form (17) with $a=5 \mu$. Other parameters used in this numerical calculation are $\varepsilon_{\mathrm{so}}=$ $0.1 \mu, \Delta=0.05 \mu, B=0.275 \mu$. The solid blue curve corresponds to Eq. (13) of the main text.

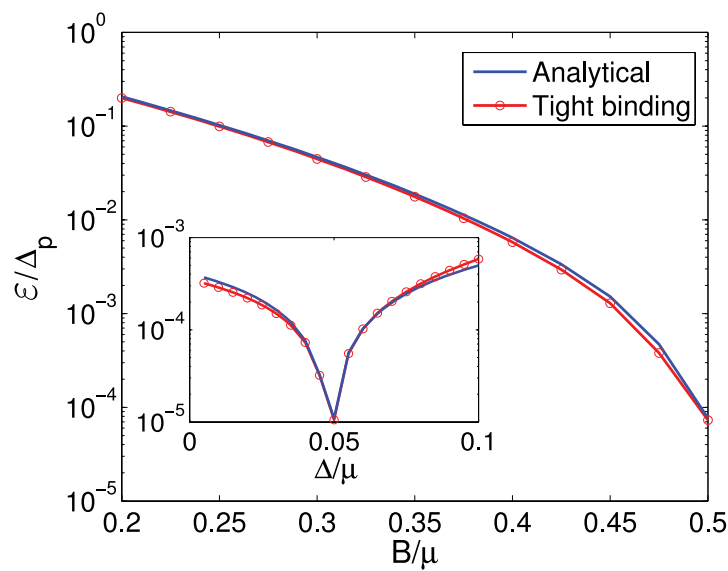

FIG. 2. (Color online) Andreev end-state energy $\varepsilon$ as a function of the Zeeman energy $B$ (main panel) and induced superconducting gap parameter $\Delta$ (inset). The parameters of the numerical calculation are $\varepsilon_{\text {so }}=0.1 \mu, \Delta=0.04 \mu$ (main figure), $B=0.5 \mu$ (inset). The confining potential has the form (17) with $a=5 \mu$ and $\sigma=1.273\left(h / p_{\mathrm{F}}\right)$.

couple the two Majorana modes for the two spin channels. This near degeneracy will be lifted in the presence of perturbations with an abrupt spatial dependence that couple the different spin-orbit bands. Examples of such perturbations are scattering from pointlike impurities (which couple left-moving and right-moving particles), or the abrupt vanishing of the pairing potential, which happens, e.g., if not all of the semiconducting wire is covered with the superconducting contact. The Andreev end-state energy $\varepsilon$ in the presence of a point impurity with potential $U \delta\left(x-x_{\mathrm{i}}\right) \tau_{z}$ is (to first order in $U$ )

$$
\varepsilon=\frac{4 U \alpha m e^{-2 \int_{x_{0}}^{x_{\mathrm{i}}} d x \xi^{-1}(x)}}{\Omega \sqrt{B^{2}+\varepsilon_{\mathrm{so}}\left(x_{\mathrm{i}}\right)^{2}}}\left|\sin \left[4 \eta+\int_{x_{0}}^{x_{\mathrm{i}}} d x \frac{m v(x)}{\hbar}\right]\right| .
$$

For the example of a slowly varying potential $V(x)=\mu-x V^{\prime}$ with a linear dependence on position, this gives

$$
\begin{aligned}
\varepsilon= & 2 U m \sqrt{\frac{\alpha^{3} V^{\prime} \Delta}{\pi \hbar B^{3}}} e^{-\frac{2 m \alpha \Delta}{\hbar B}\left(x_{i}-x_{0}\right)} \\
& \times\left|\sin \left[4 \eta+\frac{2 \sqrt{2 m V^{\prime}}}{3 \hbar}\left(x_{i}-x_{0}\right)^{3 / 2}\right]\right| .
\end{aligned}
$$

In the case where the order parameter vanishes abruptly, $\Delta(x)=\Delta \Theta\left(x-x_{\mathrm{N}}\right)$ (see the inset of Fig. 3), the end-state energy can be obtained directly from Eq. (13). For the special case where the entire potential modulation occurs in the normal region, the discontinuity in $\Delta$ contributes to the end-state energy by the amount

$$
\varepsilon=\frac{2 \hbar B}{\Omega} \frac{1}{m \alpha v_{F}} \operatorname{Re} \frac{e^{2 i \int_{x_{0}}^{x_{N}} d x k_{\mathrm{m}}(x)}}{1+i k_{\mathrm{m}} \xi},
$$

where

$$
\Omega=\frac{2 \xi}{v_{F}}+4 \int_{x_{0}}^{x_{\mathrm{N}}} d x \frac{1}{v(x)}
$$

and $k_{\mathrm{m}}, \xi$, and $v_{\mathrm{F}}$ are the asymptotic values for $x>x_{\mathrm{N}}$. Figure 3 compares numerical simulations of the model (1) with and without an abrupt change in the superconducting order parameter $\Delta$. 


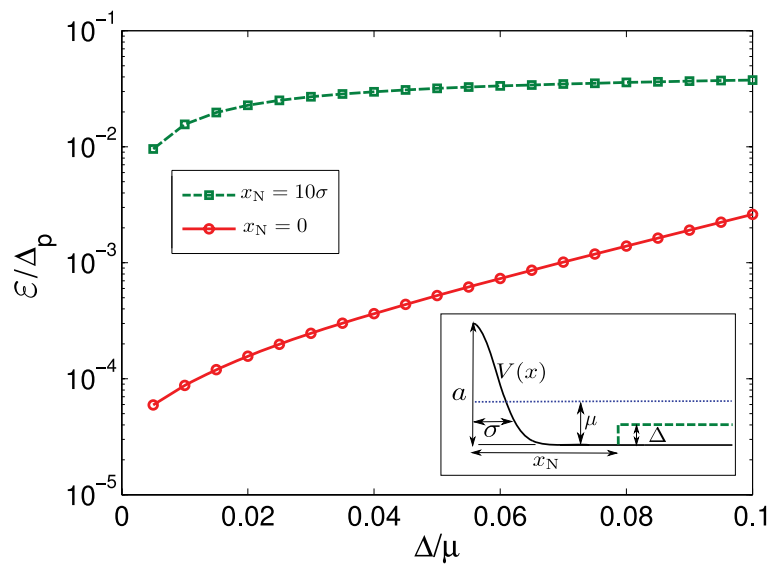

FIG. 3. (Color online) Andreev end-state energy $\varepsilon$ vs the induced superconducting gap parameter $\Delta$ with (squares) and without (circles) an abrupt termination of the order parameter $\Delta$ at position $x_{\mathrm{N}}$. The inset shows the functional form of the superconducting order $\Delta(x)=\Delta \theta\left(x-x_{\mathrm{N}}\right)$. In both cases the wire is terminated by a smooth potential $V(x)$ of the form (17) with parameters with $a=5 \mu$ and $\sigma \approx 3.183 h / p_{\mathrm{F}}$. Other parameters used in this numerical calculation are $\varepsilon_{\mathrm{so}}=0.1 \mu$ and $B=0.275 \mu$.

Up to this point, our discussion has focused on onedimensional semiconductor wires with a single transverse channel. Our arguments continue to be valid for multichannel wires. In this case, for $B \gtrsim \Delta$ each transverse channel is in a separate effectively spinless $p$-wave superconducting state. Hard-wall boundary conditions at the wire's end couple the channels, which gaps out the end states, up to the possible exception of a single Majorana end state if the total number of channels $N$ (counting spin) is odd. In general, a coupling exists between spin-degenerate channels with the same transverse mode, as well as between channels with different transverse modes, although the "off-diagonal" coupling is small if the wire width is much smaller than the superconducting coherence length $\xi$ because of an approximate chiral symmetry. ${ }^{20,29}$ By the mechanism discussed above, a smooth confinement strongly reduces the diagonal and the off-diagonal couplings between channels, giving rise to $\operatorname{int}(N / 2)$ low-energy fermionic states at the wire's end. This is illustrated in Fig. 4 for the case of a semiconducting wire with two spin-degenerate transverse channels.

In conclusion, we have shown that a smooth confining potential at the wire's ends leads to the existence of lowenergy Andreev end states even in the topologically trivial phase of one-dimensional proximity-coupled nanowires. The presence of such low-energy Andreev states would give rise to (near-) zero-bias conductance peaks deep in the topologically

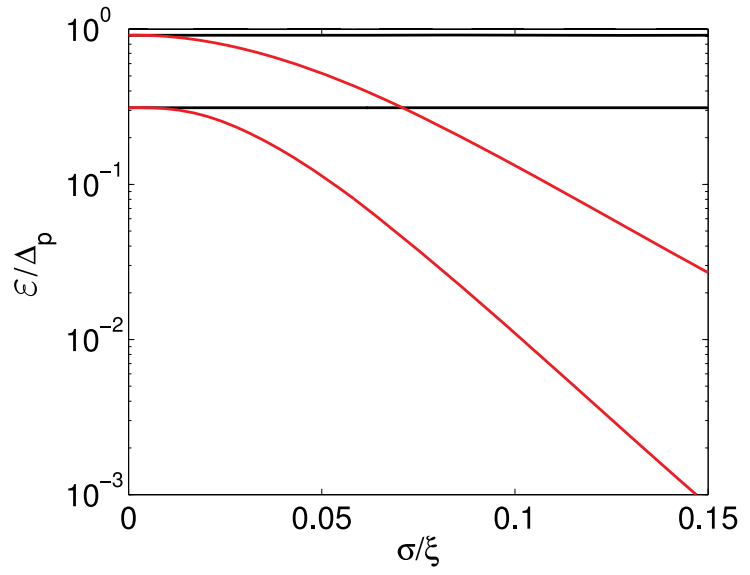

FIG. 4. (Color online) Andreev end-state energies $\varepsilon$ for a twodimensional wire with two transverse channels, normalized by the bulk excitation gap, as a function of the adiabaticity parameter $\sigma$. The Hamiltonian is given by the two-dimensional extension of Eq. (1), which has the spin-orbit coupling term $\alpha p_{x} \sigma_{y} \tau_{z}-\alpha p_{y} \sigma_{x}$. Parameters in the numerical calculation are $B=0.1667 \mu$, and wire width $W=$ $1.225 h / p_{\mathrm{F}}=0.054 \xi$. The spin-orbit energies of the two transverse bands (calculated for $B=0$ ) are $\varepsilon_{\mathrm{so}, 1}=0.074 \mu, \varepsilon_{\mathrm{so}, 2}=0.042 \mu$.

trivial parameter regime. Our findings could be relevant for recent experiments, ${ }^{11,12}$ in which the confinement at the nanowire ends is gate induced, since they provide an alternative mechanism for a zero-bias peak that needs to be ruled out in order to unambiguously identify the observed zero-bias conductance peak with a topological superconducting phase. [We note that even if $\Delta$ vanishes abruptly near the wire's end before the termination by a smooth gate-induced potential sets in, the energy of the Andreev end state in the topologically trivial phase can still be parametrically small in the limit $B \gg \varepsilon_{\text {so }}$-see Eq. (20).] At elevated temperatures, where the zero-bias peak in the tunneling density of states is thermally broadened, a Majorana bound state cannot be separated from an Andreev bound state with energy $\varepsilon \ll k_{B} T$ as the origin of the peak. One way to experimentally distinguish the two scenarios is to go to lower temperatures, where a single Majorana end state leads to a quantized conductance peak of height $2 e^{2} / h,{ }^{13,14}$ whereas a near-zero-energy Andreev bound state of the type discussed here appears as a peak of height $4 e^{2} / h$, in the limit that the zero-temperature peak width exceeds $\varepsilon$.

We gratefully acknowledge discussions with J. Danon, F. Von Oppen, Y. Oreg, and F. Pientka. This work is supported by the Alexander von Humboldt Foundation in the framework of the Alexander von Humboldt Professorship, endowed by the Federal Ministry of Education and Research.
${ }^{1}$ A. Y. Kitaev, Phys. Usp. 44, 131 (2001).

${ }^{2}$ G. Moore and N. Read, Nucl. Phys. B 360, 362 (1991).

${ }^{3}$ N. Read and D. Green, Phys. Rev. B 61, 10267 (2000).

${ }^{4}$ D. A. Ivanov, Phys. Rev. Lett. 86, 268 (2001).

${ }^{5}$ A. Kitaev, Ann. Phys. 303, 2 (2003).
${ }^{6}$ M. H. Freedman, Proc. Natl. Acad. Sci. USA 95, 98 (1998).

${ }^{7}$ A. Kitaev, Ann. Phys. 321, 2 (2006).

${ }^{8}$ C. Nayak, S. H. Simon, A. Stern, M. Freedman, and S. Das Sarma, Rev. Mod. Phys. 80, 1083 (2008). 
${ }^{9}$ Y. Oreg, G. Refael, and F. von Oppen, Phys. Rev. Lett. 105, 177002 (2010).

${ }^{10}$ R. M. Lutchyn, J. D. Sau, and S. Das Sarma, Phys. Rev. Lett. 105, 077001 (2010).

${ }^{11}$ V. Mourik, K. Zuo, S. M. Frolov, S. R. Plissard, E. P. A. M. Bakkers, L. P. Kouwenhoven, Science 336, 1003 (2012).

${ }^{12}$ A. Das, Y. Ronen, Y. Most, Y. Oreg, M. Heiblum, and H. Shtrikman, arXiv:1205.7073.

${ }^{13}$ K. T. Law, P. A. Lee, and T. K. Ng, Phys. Rev. Lett. 103, 237001 (2009).

${ }^{14}$ K. Flensberg, Phys. Rev. B 82, 180516(R) (2010).

${ }^{15}$ M. Wimmer, A. R. Akhmerov, M. V. Medvedyeva, J. Tworzydlo, and C. W. J. Beenakker, Phys. Rev. Lett. 105, 046803 (2010).

${ }^{16}$ A. C. Potter and P. A. Lee, Phys. Rev. Lett. 105, 227003 (2010).

${ }^{17}$ R. M. Lutchyn, T. D. Stanescu, and S. Das Sarma, Phys. Rev. Lett. 106, 127001 (2011).

${ }^{18}$ A. C. Potter and P. A. Lee, Phys. Rev. B 83, 184520 (2011); 84, 059906(E) (2011).
${ }^{19}$ T. D. Stanescu, R. M. Lutchyn, and S. Das Sarma, Phys. Rev. B 84, 144522 (2011).

${ }^{20}$ G. Kells, D. Meidan, and P. W. Brouwer, Phys. Rev. B 85, 060507(R) (2012).

${ }^{21}$ A. C. Potter and P. A. Lee, Phys. Rev. B 85, 094516 (2012).

${ }^{22}$ M. Gibertini, F. Taddei, M. Polini, and R. Fazio, Phys. Rev. B 85, 144525 (2012).

${ }^{23}$ S. Tewari, T. D. Stanescu, J. D. Sau, and S. Das Sarma, Phys. Rev. B 86, 024504 (2012).

${ }^{24}$ M.-T. Rieder, G. Kells, M. Duckheim, D. Meidan, and P. W. Brouwer, arXiv:1207.2993.

${ }^{25}$ J. Liu, A. C. Potter, K. T. Law, and P. A. Lee, arXiv:1206.1276.

${ }^{26}$ D. I. Pikulin, J. P. Dahlhaus, M. Wimmer, H. Schomerus, and C. W. J. Beenakker, arXiv:1206.6687.

${ }^{27}$ D. Bagrets and A. Altland, arXiv:1206.0434.

${ }^{28}$ E. Prada, P. San-Jose, and R. Aguado, arXiv:1203.4488.

${ }^{29}$ S. Tewari and J. D. Sau, arXiv:1111.6592. 